\title{
Hybridization, Inter-Ion Correlation, and Surface States in the Kondo Insulator $\mathrm{SmB}_{6}$
}

\author{
Xiaohang Zhang, ${ }^{1}{ }^{*}$ N. P. Butch, ${ }^{2}$ P. Syers, ${ }^{1}$ S. Ziemak, ${ }^{1}$ Richard L. Greene, ${ }^{1}$ and Johnpierre Paglione ${ }^{1}$ \\ ${ }^{1}$ Center for Nanophysics and Advanced Materials \& Department of Physics, \\ University of Maryland, College Park, Maryland 20742, USA \\ ${ }^{2}$ Condensed Matter and Materials Division, Lawrence Livermore National Laboratory, Livermore, California 94550, USA
}

(Received 24 November 2012; revised manuscript received 15 January 2013; published 14 February 2013)

As an exemplary Kondo insulator, $\mathrm{SmB}_{6}$ has been studied for several decades. However, direct evidence for the development of the Kondo coherent state and for the evolution of the electronic structure in the material has not been obtained due to the compound's rather complicated electronic and thermal transport behavior. Recently, these open questions have attracted increasing attention as the emergence of a time-reversal-invariant topological surface state in the Kondo insulator has been suggested. Here, we use point-contact spectroscopy to reveal the temperature dependence of the electronic states in $\mathrm{SmB}_{6}$. We demonstrate that $\mathrm{SmB}_{6}$ is a model Kondo insulator: Below $100 \mathrm{~K}$, the conductance spectra reflect the Kondo hybridization of $\mathrm{Sm}$ ions, but, below about $30 \mathrm{~K}$, signatures of inter-ion correlation effects clearly emerge. Moreover, we find evidence that the low-temperature insulating state of this exemplary Kondo-lattice compound harbors conduction states on the surface, in support of predictions of nontrivial topology in Kondo insulators.

DOI: 10.1103/PhysRevX.3.011011

The many-body screening interaction between a single localized spin and a continuum of conduction electrons known as the Kondo effect is a well-established element of many physical systems, such as noble metals [1], quantum dots [2], and graphene [3], etc. When spins are arranged in a periodic array, called a Kondo lattice, the hybridization leads further to the characteristic opening of an energy gap in the electronic density of states [4]. However, this signature in the electronic structure has not yet been unambiguously observed in Kondo insulators.

Unlike conventional band insulators, a Kondo insulator (KI) features an energy gap in the electronic density of states (DOS) whose magnitude is strongly temperature dependent and only fully developed at low temperatures. This sensitivity to temperature is rooted in the electronic interactions underlying the Kondo effect. At high temperatures, localized spins on ions are only weakly coupled to the conduction electrons in their host material. As the temperature is lowered below a material-dependent characteristic Kondo temperature, the localized spin states hybridize with the itinerant electrons, forming a manybody spin-singlet state. When considering only a single isolated ion, the preceding description is enough, but, in a Kondo lattice, the proximity of periodic ions brings into play additional correlations on further cooling, resulting in the reconstruction of the electronic band structure and the

\footnotetext{
*Present Address: National Institute of Standards and Technology, Gaithersburg, MD 20899, USA

Published by the American Physical Society under the terms of the Creative Commons Attribution 3.0 License. Further distribution of this work must maintain attribution to the author(s) and the published article's title, journal citation, and DOI.
}

formation of a hybridization gap in the DOS [4]. The KI is a special case of the Kondo lattice, in which the Fermi level or chemical potential falls in the gap [Fig. 1(b)], in contrast to heavy-fermion metals [5], in which the Fermi level coincides with a finite (and large) DOS.

Although this phenomenology is widely accepted, the experimental evidence for the temperature evolution of the electronic states in these complex systems is incomplete. An ideal tool to address the issue is quasiparticle tunneling spectroscopy, which directly probes the DOS. In the more straightforward case of a single-ion Kondo system, the conductance spectrum reflects the interference between the two paths that an injected electron can take [Fig. 1(a)]: one directly to the itinerant electrons and the other indirectly through the hybridized many-body Kondo state. This interference causes a characteristic Fano-resonance line shape [6], as first observed in scanning-tunnelingspectroscopy (STS) measurements on single magnetic adatoms embedded in normal metals [7].

In a Kondo lattice, the development of the correlated ground state should substantially modify the Fano line shape [8-18]. However, these effects have not been clearly observed in STS [13-16] and point-contact-spectroscopy (PCS) $[17,18]$ measurements on heavy-fermion metals. It is possible that signatures of correlation effects may be clouded by the presence of competing interactions underlying tendencies toward superconductivity, magnetism, or quantum criticality [4,5]. For example, substantial differences in the detailed characteristics and the temperature dependence of the conductance spectra obtained on $\mathrm{URu}_{2} \mathrm{Si}_{2}[13,14,18]$ hold different implications for the relationship between the Kondo ground state and the mysterious hidden-order phase. In contrast, Kondo insulators, 


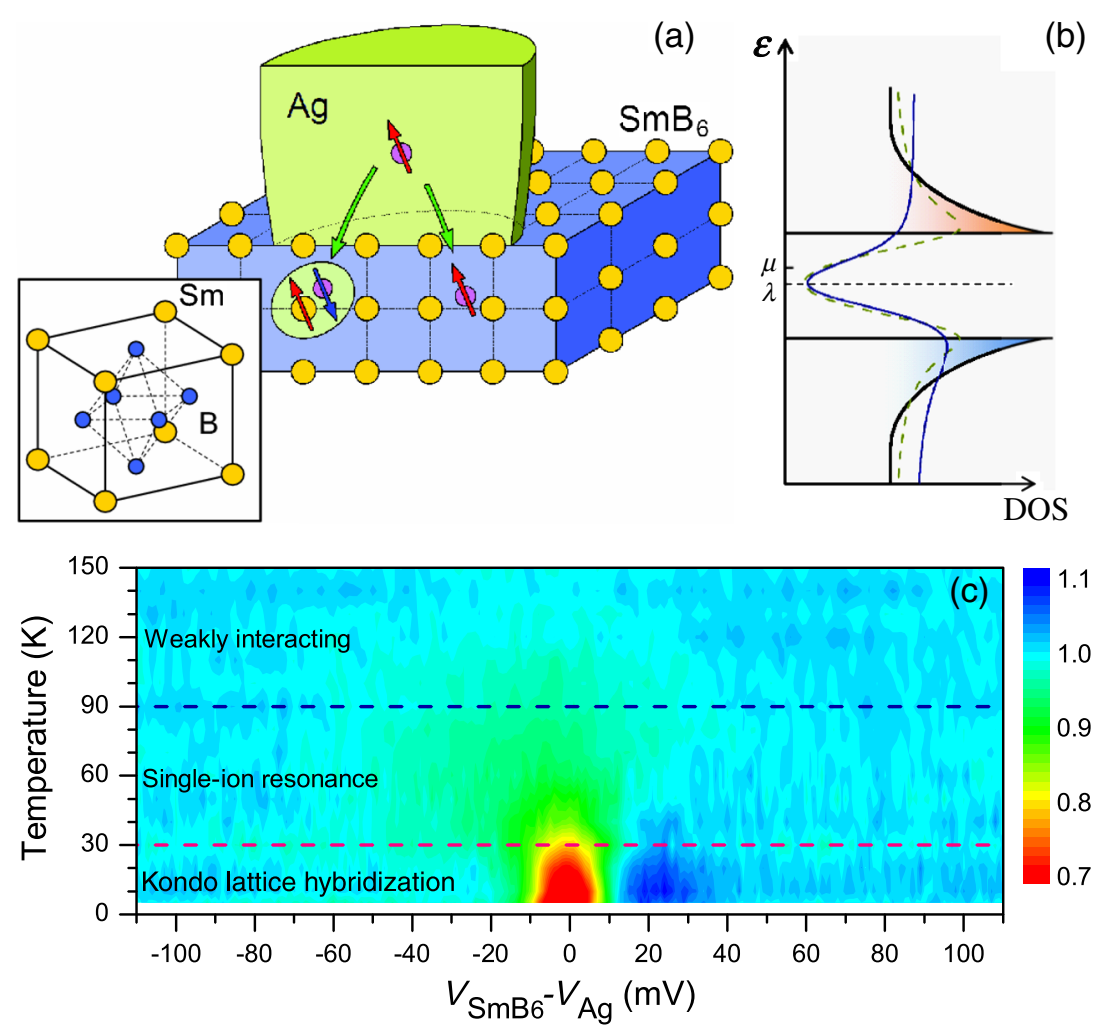

FIG. 1. (a) A schematic view for a Ag-Sm 6 junction. A Fano-like conductance spectrum is expected due to the interference of the two electron paths, as indicated by the two green arrows: One leads directly into the itinerant electron states (right side), and thus should reflect the DOS. The other leads indirectly into the electron sea through a spin-singlet Kondo state (left side). Inset: The crystal structure of the $\mathrm{SmB}_{6}$ single crystal. The $\mathrm{Sm}$ ions form a simple cubic structure. (b) A schematic view of the DOS for the ground state of $\mathrm{SmB}_{6}$. In point-contact-spectroscopy measurement, quasiparticle broadening should be taken into account, as indicated by the green dashed line. The blue solid curve is the expected spectrum with Fano resonance. $\mu$ is the chemical potential while $\lambda$ is the renormalized $f$ level. (c) The contour map of the normalized conductance in the bias voltage-temperature $(V-T)$ plane. All conductance data are normalized by the spectrum conductance obtained at $150 \mathrm{~K}$. As indicated by the color legend, red and blue colors represent values below 0.7 and above 1.1, respectively. At temperatures above about $90 \mathrm{~K}$, the conductance spectroscopy of the junction is highly symmetric, with negligible temperature dependence due to the weak interaction between electrons and local moments. From $90 \mathrm{~K}$ to $30 \mathrm{~K}$, the zero-bias conductance is gradually suppressed, while an asymmetric conductance line shape develops. In this temperature range, the conductance spectrum can be described by the single-ion resonance model. Below about $30 \mathrm{~K}$, the conductance spectra are clearly asymmetric with a dip at zero bias and a peak at $20 \mathrm{mV}$. As discussed in the main text, this strongly asymmetric conductance line shape provides direct evidence for the inter-ion correlations in the Kondo lattice system.

which lack competing ordered phases, make an attractive test bed for the study of electronic interactions in Kondo lattices.

One such material is $\mathrm{SmB}_{6}$, which crystallizes as a simple cubic lattice of samarium ions separated by boron octahedra [inset of Fig. 1(a)]. The basic ingredients of local-itinerant hybridization have long been recognized: The electronic configuration of the $\mathrm{Sm}$ ions fluctuates between $4 f^{6}\left(\mathrm{Sm}^{2+}\right)$ and $4 f^{5} 5 d\left(\mathrm{Sm}^{3+}\right)$, giving an effective valence of 2.5 at low temperatures $[19,20]$; there is a 20-meV gap in the optical conductivity [21]; the lowtemperature magnetic susceptibility is indicative of a nonmagnetic ground state [22]; the temperature dependence of the electrical resistivity is insulating, increasing by several orders of magnitude on cooling [23], while the carrier density decreases to $10^{17} \mathrm{~cm}^{-3}$ [24]. Yet the validity of the Kondo insulator description for $\mathrm{SmB}_{6}$ is not agreed on $[23,25-28]$. One point of particular concern has been that there is finite electrical resistivity at very low temperatures, whereas, in a fully gapped insulator, it should be infinite [25-27]. Our new measurements clarify this issue, showing that $\mathrm{SmB}_{6}$ acts like a model Kondo insulator [8], and the absence of true insulating behavior at low temperatures reflects the influence of surface conducting states [29].

Point-contact spectroscopy is a versatile tool for the study of the electronic structure in various materials, including superconductors [30,31] and ferromagnets [32,33]. It has been demonstrated that the point-contact-spectroscopy technique can be used to probe the bulk DOS and study the manybody interactions in strongly correlated materials ([18,34]; also see Part I of Ref. [35]). Previous measurements on $\mathrm{SmB}_{6}[36-39]$ have reported an asymmetric line shape with 
gaplike features in the low-temperature point-contact spectroscopy, but definitive explanations of the shape and the temperature evolution are lacking. In our study, the temperature dependence of the differential point-contact conductance data, $d I / d V$, measured using a $\mathrm{Ag}-\mathrm{SmB}_{6}$ junction, is summarized in Fig. 1(c). Below $100 \mathrm{~K}$, the zero-bias conductance starts to decrease, forming a trough with a half-width of about $20 \mathrm{mV}$ at low temperatures [Fig. 2(a)]. The emergence of this feature reflects the onset of Kondo hybridization between the Sm local moments and the conduction electrons and is consistent with other properties. At a similar temperature, the magnetic susceptibility deviates from high-temperature independent-spin paramagnetism, leading to a broad hump at lower
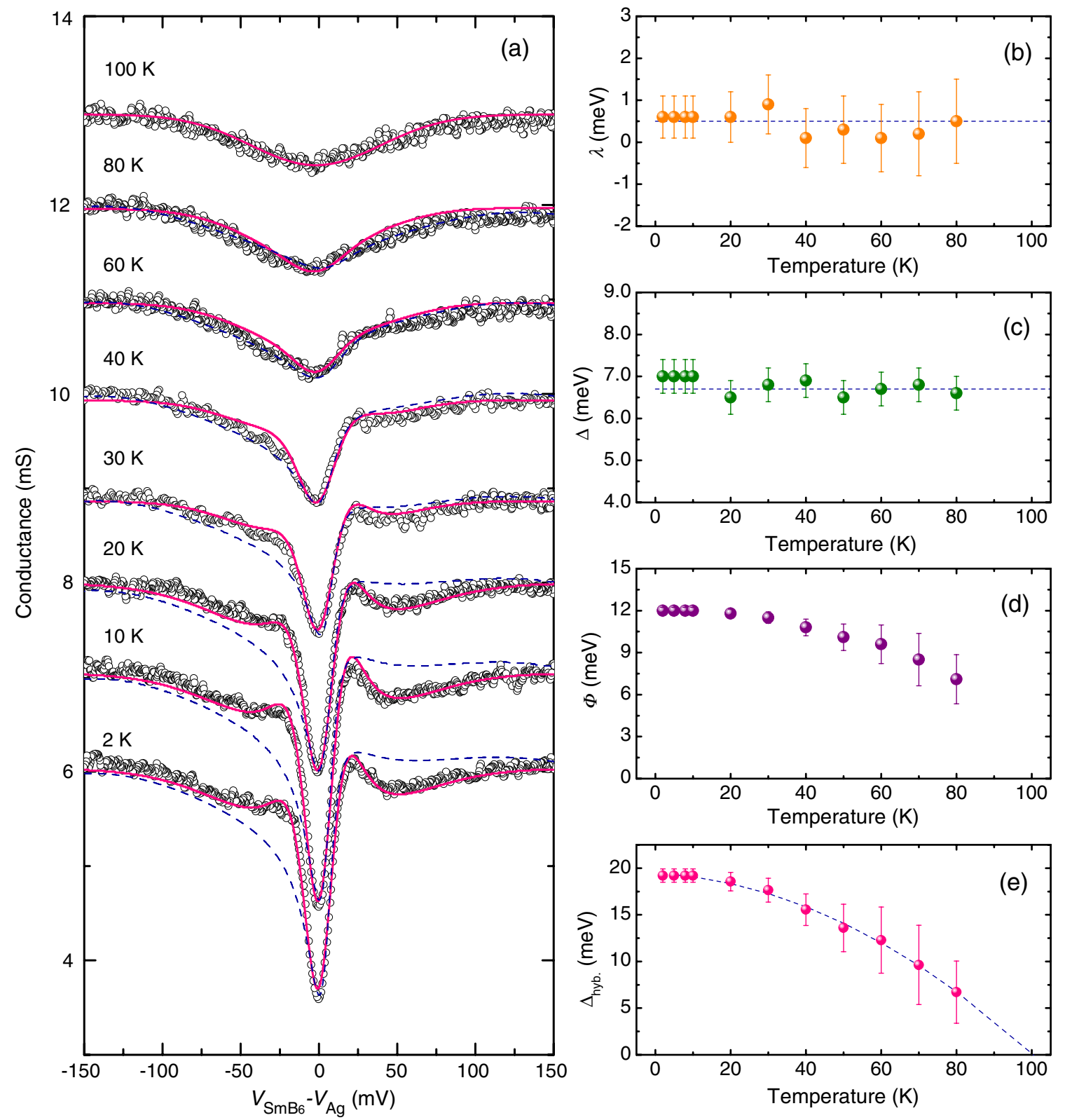

FIG. 2. (a) The observed conductance spectra with the best fits to the classical Fano-resonance line shape [Eq. (1) and dashed lines] and to the tunneling model for Kondo lattices [Eq. (2) and solid lines], respectively. For clarity, only the spectrum and the simulations for $2 \mathrm{~K}$ are plotted with the actual value, while other curves are vertically shifted. (b), (c), and (d) show the temperature dependence of $\lambda$ (renormalized $f$ level), $\Delta$ (Kondo resonance width), and $\Phi$ (half of the direct gap size), respectively, used in the Kondo-latticetunneling simulations. The dashed line in (b) and (c) suggests that both the renormalized $f$ level and the Kondo gap width are nearly temperature independent in the entire temperature range below $100 \mathrm{~K}$. (e) The temperature dependence of the hybridization gap extracted from the hybridization amplitude and the bandwidth $D$, based on the Kondo-lattice-tunneling model. The dashed line in (e) represents a smooth trend of the size of the hybridization gap as the temperature changes. The extrapolation of the curve suggests that the hybridization gap vanishes at a temperature of around $100 \mathrm{~K}$. 
temperatures [22]. X-ray-absorption-spectroscopy studies also show a substantial decrease in Sm valence, starting at about $100 \mathrm{~K}$ [19]. In addition, angle-resolved photoemission measurements indicate that a gap in the DOS opens at about $100 \mathrm{~K}$ [28]. As temperature decreases, the pointcontact conductance spectrum develops an asymmetry that becomes especially pronounced below $40 \mathrm{~K}$, where a conductance peak clearly appears at $20 \mathrm{mV}$. Although the relationship between similar spectroscopy features and Kondo hybridization was previously questioned [38], on quantitative examination of our conductance spectra, it becomes clear that the asymmetric peak-trough structure matches theoretical predictions for tunneling into Kondo lattices [8-12].

Beginning with the classical Fano spectral line shape [6], the differential conductance is given by

$$
G(V) \propto \frac{\left[\left(e V-E_{0}\right) / \Gamma+q\right]^{2}}{1+\left[\left(e V-E_{0}\right) / \Gamma\right]^{2}},
$$

where $E_{0}$ is the Kondo resonance energy, $\Gamma$ is the full width at half maximum, and $q$ is the Fano factor, which reflects the ratio of probabilities between the two tunneling paths. The best Fano-resonance fits (see Part IV of Ref. [35]) are presented as dashed curves in Fig. 2(a). Clearly, the classical Fano model provides a quantitative description for the development of the zero-bias dip as well as for the asymmetric conductance line shape as temperature decreases. In particular, the simulations captured well the conductance spectra at temperatures in a broad range from 100-30 K. Moreover, the temperature dependence of the fitting parameters (see Part IV of Ref. [35]) establishes a phenomenological understanding for the asymmetric point-contact spectroscopy in the framework of Kondo resonance. Despite the great success in applying the classical Fano model, we notice that the original Fano-resonance line shape fails to precisely describe the conductance spectra below $30 \mathrm{~K}$, or once the asymmetric peak structure at $20 \mathrm{mV}$ becomes evident.

It is important to note that the classical Fano line shape [Eq. (1)] explains the tunneling spectrum of single magnetic impurity systems, in which the DOS is temperature independent and the thermal evolution of the spectrum is solely attributed to the Kondo resonance. In contrast, the local moments in Kondo lattices are strongly coupled to each other, and the collective interactions result in a reconstruction of the electronic DOS that must be simultaneously considered. Indeed, recent studies on heavyfermion metals $[15,18]$ have also suggested the inadequacy of the classical Fano analysis.

To account for the evolution of the electronic structure in Kondo lattices, Maltseva et al. [8] calculated the tunneling current based on the large- $N$ mean-field theory. The differential tunneling conductance obtained in the study is given by

$$
\begin{aligned}
G(V) \propto & \operatorname{Im} \tilde{G}_{\psi}^{\mathrm{KL}}(e V) ; \\
\tilde{G}_{\psi}^{\mathrm{KL}}(e V)= & \left(1+\frac{q \Delta}{e V-\lambda}\right)^{2} \ln \left(\frac{e V+D_{1}-\frac{\Phi^{2}}{e V-\lambda}}{e V-D_{2}-\frac{\Phi^{2}}{e V-\lambda}}\right) \\
& +\frac{2 D / t_{C}^{2}}{e V-\lambda},
\end{aligned}
$$

where $-D_{1}$ and $D_{2}$ are, respectively, the lower and the upper conduction band edges, $2 D=D_{1}+D_{2}$ is the bandwidth, $\Delta$ is the width of the Kondo resonance, $\lambda$ is the renormalized $f$ level, and $2 \Phi$ is the direct gap in momentum space. The Fano factor $q=\left(\Phi t_{f}\right) /\left(\Delta t_{c}\right)$, where $t_{f}$ and $t_{c}$ are the matrix amplitudes for tunneling into the Kondo states and into the itinerant electrons, respectively. Finally, the indirect hybridization gap $\Delta_{\text {hyb }}$ is given by $2 \Phi^{2} / D$. To represent the quasiparticle broadening effect, a $\gamma_{0}$ term is introduced [40]. As a consequence, the energy, $e V$, in Eq. (2) is replaced by $e V-i \gamma_{0}$. With a background representing the DOS at high temperatures, the best fits (see Part V in Ref. [35]) to our experimental data based on Eq. (2) are shown as solid lines in Fig. 2(a). The results indicate a nearly constant $\lambda$ term of about $0.5 \mathrm{meV}$ in the whole temperature range [Fig. 2(b)], suggesting that the renormalized $f$ level is pinned slightly above the Fermi level of the Kondo insulator. The simulations also result in a constant Kondo resonance width of about $6.7 \mathrm{mV}$ [Fig. 2(c)], which is consistent with the scale of the Kondo temperature (about $80 \mathrm{~K}$ ). Fig. 2(e) shows the temperature dependence of the hybridization gap calculated from the hybridization amplitude [Fig. 2(d)] and the bandwidth. At temperatures below $30 \mathrm{~K}$, the hybridization gap retains a constant value of about $18 \mathrm{meV}$, which is consistent with the gap size determined by studies of lowtemperature resistivity [23,41], specific heat [42], and optical reflectivity [21]. The hybridization gap decreases at higher temperatures and completely vanishes at a temperature of around $100 \mathrm{~K}$.

The remarkable agreement between our experimental data and the model of Maltseva et al.. [Eq. (2)] [8] provides the first direct evidence for the simultaneous development of the Kondo coherent state and the hybridization gap in a Kondo insulator. Based on the temperature range (30-40 K) where the classical Fano line shape fails to adequately describe the data, we conclude that correlation effects and the ensuing change in the DOS play a physically significant role at surprisingly high temperatures. In fact, in this temperature range, the electrical resistivity increases dramatically, and the Hall voltage changes sign [24], which links the telltale characteristics of $\mathrm{SmB}_{6}$ convincingly to Kondo correlation effects.

One unaddressed question remains, however, and it is central to the controversy surrounding the proper description of $\mathrm{SmB}_{6}$ : Why does the resistivity not diverge but rather become temperature independent below 4 K [FIG. 3(a)]? The answer has typically been that the behavior is due to 


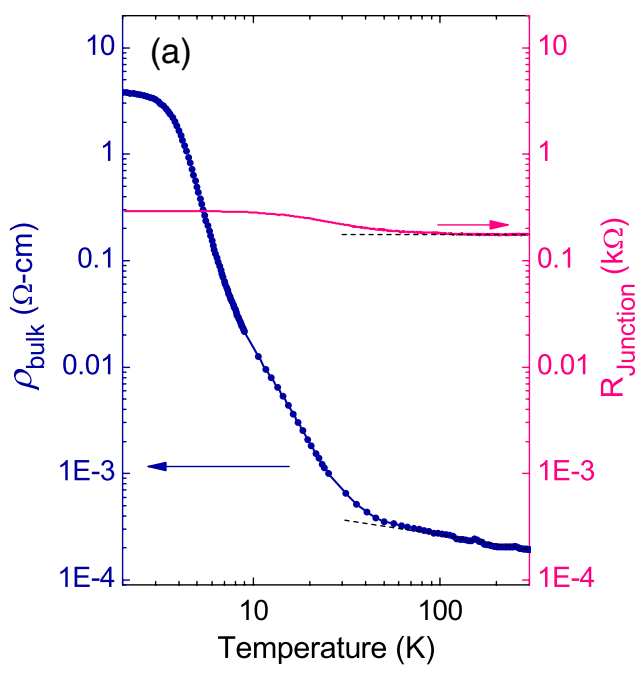

(b)

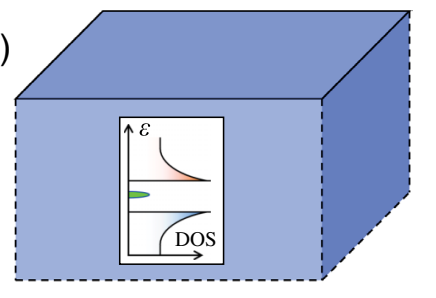

(c)

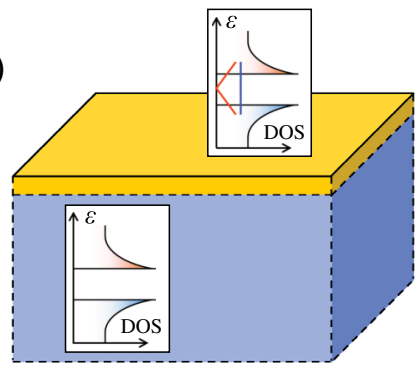

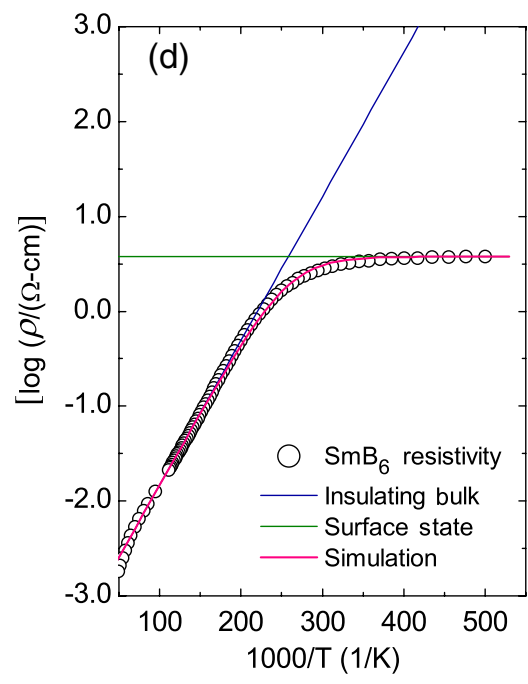

FIG. 3. (a) Temperature dependence of electrical resistivity of a $\mathrm{SmB}_{6}$ single crystal (blue data points) and the resistance of a $\mathrm{Ag}-\mathrm{SmB}_{6}$ junction (pink). Compared to the 4 order-of-magnitude increase in the resistivity and a significant suppression below $4 \mathrm{~K}$ for single $\mathrm{SmB}_{6}$ crystals, the temperature dependence of the $\mathrm{Ag}-\mathrm{SmB}_{6}$ junction resistance exhibits a rather different behavior with an overall increase of about 2 in the measured temperature range and a low-temperature saturation below about $10 \mathrm{~K}$. The dashed lines indicate that the junction resistance holds a constant value at temperatures above about $10 \mathrm{~K}$, while the resistivity of bulk single crystals shows an activation behavior in the same temperature range. (b) Schematic view of the DOS in $\mathrm{SmB}_{6}$ without the presence of a surface state. It is expected that the coherent transport of the bulk in-gap bound states (green area) leads to the suppression in resistivity at temperatures below $4 \mathrm{~K}$. (c) The DOS in $\mathrm{SmB}_{6}$ with the emergence of a surface state at low temperatures. The finite residue resistivity of the material is due to the presence of a conducting surface state. Both topological surface states (orange) and conventional two-dimensional electron gas (blue) could lead to such a metallic 2D conduction. (d) Simulation of the measured resistivity in the scenario that a metallic-like surface state dominates the conductance at low temperatures. The overall conductivity is the sum of two conduction channels: the surface conduction (green) and the intrinsic bulk conduction (blue). The measured resistivity approaches the saturation point at a temperature of around $4 \mathrm{~K}$.

in-gap states [43] caused by either impurities, incomplete hybridization, or some more exotic scenario. There is surprisingly little direct evidence that any in-gap bound states exist. Claims of a 3.5-meV activation energy are based on fits to resistivity data from $5-50 \mathrm{~K}$, specifically in a temperature range at which the hybridization gap is temperature dependent and activated behavior would not be expected.

A solution to this conundrum was suggested recently by Dzero et al. in their groundbreaking proposal of topological Kondo insulators [29], where $\mathrm{SmB}_{6}$ was singled out as the best candidate for realizing a strong topological insulator. It is widely believed that the unusual residual resistivity results from the presence of a metallic in-gap conduction channel at low temperatures, and candidate states have been proposed to exist in the bulk or on the surface [Figs. 3(b) and 3(c)]. Our spectroscopic measurements of the bulk Kondo-insulator state allow us to determine which of these scenarios is correct. If the saturation of the bulk resistivity were due to the coherent transport of bulk in-gap states [20,41,43], the electronic structure would change at about $4 \mathrm{~K}$ as a coherent conduction channel develops. In terms of spectroscopy, the enhanced DOS at the Fermi level would lead to a zero-bias peak in the point-contact-spectroscopy measurement, or, at the very least, excess spectral weight. Instead, our results suggest that the bulk DOS is stable and constant in the entire temperature range below $10 \mathrm{~K}$. Thus we rule out the presence of bulk in-gap states. It is clear then that the temperature dependence of the electrical resistivity must reflect a competition between the true Kondo insulator in the bulk and a metallic surface state. Within this picture, the resistivity of $\mathrm{SmB}_{6}$ crystals reflects the activated behavior due to the presence of the hybridization gap in the bulk [20,43], except at the lowest temperatures, where surface conduction becomes predominant. As shown in Fig. 3(d), a simple two-channel transport simulation based on this picture clearly indicates that the bulk resistivity approaches a saturation point at about $4 \mathrm{~K}$. Despite the success of this explanation, we note that the topological character of the surface metallic states has yet to be determined. For example, the presence of topologically trivial surface electronic states might arise from electronic or structural surface reconstruction, as possibly exemplified by a higher $\mathrm{Sm}^{3+} / \mathrm{Sm}^{2+}$ ratio at the surface of $\mathrm{SmB}_{6}$ [44]. To address this critical question, surface-dominant tunneling-spectroscopic measurements are being pursued.

The authors acknowledge fruitful discussions with A. Nicolaou, J. Zhou, G. Levy, and A. Damascelli at the University of British Columbia, V. Galitski at the University of Maryland, and M. Dzero at Kent State 
University. We especially thank Laura H. Greene of the University of Illinois at Urbana-Champaign for her thoughtful comments and suggestions. X.Z. and N.P. B. also thank K. Jin, S. R. Saha, and R. W. Hu for technical assistance. The work at the University of Maryland was supported in part by the NSF under Grant Nos. DMR-1104256 and DMR-0952716 and the AFOSR-MURI under Grant No. FA9550-09-1-0603.

Note added.-During the review of this manuscript, two preprints $[45,46]$ have given transport evidence for a conducting surface state in $\mathrm{SmB}_{6}$ at low temperatures. This evidence is a direct confirmation of the conclusions reported here of our point-contact-spectroscopy studies of $\mathrm{SmB}_{6}$.

[1] J. Kondo, Resistance Minimum in Diluted Magnetic Alloys, Prog. Theor. Phys. 32, 37 (1964).

[2] D. Goldhaber-Gordon, H. Shtrikman, D. Mahalu, D. Abusch-Magder, U. Meirav, and M. A. Kastner, Kondo Effect in a Single-Electron Transistor, Nature (London) 391, 156 (1998).

[3] J.-H. Chen, L. Li, W. G. Cullen, E. D. Williams, and M. S. Fuhrer, Tunable Kondo Effect in Graphene with Defects, Nat. Phys. 7, 535 (2011).

[4] See the review by P. Coleman, Fundamentals and Theory, edited by H. Kronmüller and S. S. P. Parkin, Handbook of Magnetism and Advanced Magnetic Materials, Vol. 1 (John Wiley, New York, 2007), pp. 95-148.

[5] A. C. Hewson, The Kondo Problem to Heavy Fermions, Cambridge Studies in Magnetism, edited by D. Edwards and D. Melville (Cambridge University Press, Cambridge, England, 1993).

[6] U. Fano, Effects of Configuration Interaction on Intensities and Phase Shifts, Phys. Rev. 124, 1866 (1961).

[7] For example, V. Madhavan, W. Chen, T. Jamneala, M. F. Crommie, and N.S. Wingreen, Tunneling into a Single Magnetic Atom: Spectroscopic Evidence of the Kondo Resonance, Science 280, 567 (1998).

[8] M. Maltseva, M. Dzero, and P. Coleman, Electron Cotunneling into a Kondo Lattice, Phys. Rev. Lett. 103, 206402 (2009).

[9] Y.-F. Yang, Fano Effect in the Point Contact Spectroscopy of Heavy-Electron Materials, Phys. Rev. B 79, 241107(R) (2009).

[10] J. Figgins and D. K. Morr, Differential Conductance and Quantum Interference in Kondo Systems, Phys. Rev. Lett. 104, 187202 (2010).

[11] P. Wölfle, Y. Dubi, and A. V. Balatsky, Tunneling into Clean Heavy Fermion Compounds: Origin of the Fano Line Shape, Phys. Rev. Lett. 105, 246401 (2010).

[12] J. X. Zhu, J. P. Julien, Y. Dubi, and A. V. Balatsky, Local Electronic Structure and Fano Interference in Tunneling into a Kondo Hole System, Phys. Rev. Lett. 108, 186401 (2012).

[13] A. R. Schmidt, M. H. Hamidian, P. Wahl, F. Meier, A. V. Balatsky, J. D. Garrett, T. J. Williams, G. M. Luke, and J. C. Davis, Imaging the Fano Lattice to 'Hidden Order'
Transition in $\mathrm{URu}_{2} \mathrm{Si}_{2}$, Nature (London) 465, 570 (2010).

[14] P. Aynajian, E. H. da Silva Neto, C. V. Parker, Y. Huang, A. Pasupathy, J. Mydosh, and A. Yazdani, Visualizing the Formation of the Kondo Lattice and the Hidden Order in $\mathrm{URu}_{2} \mathrm{Si}_{2}$, Proc. Natl. Acad. Sci. U.S.A. 107, 10383 (2010).

[15] S. Ernst, S. Kirchner, C. Krellner, C. Geibel, G. Zwicknagl, F. Steglich, and S. Wirth, Emerging Local Kondo Screening and Spatial Coherence in the HeavyFermion Metal $\mathrm{YbRh}_{2} \mathrm{Si}_{2}$, Nature (London) 474, 362 (2011).

[16] P. Aynajian, E. H. da Silva Neto, A. Gyenis, R. E. Baumbach, J. D. Thompson, Z. Fisk, E. D. Bauer, and A. Yazdani, Visualizing Heavy Fermions Emerging in a Quantum Critical Kondo Lattice, Nature (London) 486, 201 (2012).

[17] W. K. Park, J.L. Sarrao, J.D. Thompson, and L.H. Greene, Andreev Reflection in Heavy-Fermion Superconductors and Order Parameter Symmetry in $\mathrm{CeCoIn}_{5}$, Phys. Rev. Lett. 100, 177001 (2008).

[18] W. K. Park, P.H. Tobash, E. Ronning, E.D. Bauer, J.L. Sarrao, J.D. Thompson, and L.H. Greene, Observation of the Hybridization Gap and Fano Resonance in the Kondo Lattice $\mathrm{URu}_{2} \mathrm{Si}_{2}$, Phys. Rev. Lett. 108, 246403 (2012).

[19] M. Mizumaki, S. Tsutsui, and F. Iga, Temperature Dependence of $\mathrm{Sm}$ Valence in $\mathrm{SmB}_{6}$ Studied by X-Ray Absorption Spectroscopy, J. Phys. Conf. Ser. 176, 012034 (2009).

[20] T. Caldwell, A. P. Reyes, W. G. Moulton, P. L. Kuhns, M. J. R. Hoch, P. Schlottmann, and Z. Fisk, High-Field Suppression of In-Gap States in the Kondo Insulator $\mathrm{SmB}_{6}$, Phys. Rev. B 75, 075106 (2007).

[21] B. Gorshunov, N. Sluchanko, A. Volkov, M. Dressel, G. Knebel, A. Loidl, and S. Kunii, Low-Energy Electrodynamics of $\mathrm{SmB}_{6}$, Phys. Rev. B 59, 1808 (1999).

[22] S. Gabáni, K. Flachbart, V. Pavlík, T. Herrmannsdörfer, E.S. Konovalova, Yu. Paderno, J. Bednarčin, and J. Trpčevská, Magnetic Properties of $\mathrm{SmB}_{6}$ and $\mathrm{Sm}_{1-x} \mathrm{La}_{x} \mathrm{~B}_{6}$ Solid Solutions, Czech. J. Phys. 52, A225 (2002).

[23] J. C. Cooley, M. C. Aronson, Z. Fisk, and P. C. Canfield, $\mathrm{SmB}_{6}$ : Kondo Insulator or Exotic Metal?, Phys. Rev. Lett. 74, 1629 (1995).

[24] J. W. Allen, B. Batlogg, and F. Wachter, Large LowTemperature Hall Effect and Resistivity in Mixed-Valent $\mathrm{SmB}_{6}$, Phys. Rev. B 20, 4807 (1979).

[25] K. A. Kikoin and A. S. Mishchenko, Magnetic Excitations in Intermediate-Valence Semiconductors with a Singlet Ground State, J. Phys. Condens. Matter 7, 307 (1995).

[26] B. R. Coles, Speculations Concerning $\mathrm{SmB}_{6}$, Physica (Amsterdam) 230-232, 718 (1997).

[27] T. Kasuya, Physical Mechanism in Kondo Insulator, J. Phys. Soc. Jpn. 65, 2548 (1996).

[28] S. Nozawa, T. Tsukamoto, K. Kanai, T. Haruna, S. Shin, and S. Kunii, Ultrahigh-Resolution and Angle-Resolved Photoemission Study of $\mathrm{SmB}_{6}$, J. Phys. Chem. Solids 63, 1223 (2002).

[29] M. Dzero, K. Sun, V. Galitski, and P. Coleman, Topological Kondo Insulators, Phys. Rev. Lett. 104, 106408 (2010). 
[30] A. Biswas, P. Fournier, M.M. Qazilbash, V. N. Smolyaninova, H. Balci, and R. L. Greene, Evidence of $a d$ - to $s$-Wave Pairing Symmetry Transition in the Electron-Doped Cuprate Superconductor $\mathrm{Pr}_{2-x} \mathrm{Ce}_{x} \mathrm{CuO}_{4}$, Phys. Rev. Lett. 88, 207004 (2002).

[31] X. H. Zhang, Y.S. Oh, Y. Liu, L. Q. Yan, S. R. Saha, N. P. Butch, K. Kirshenbaum, K. H. Kim, J. Paglione, R. L. Greene, and I. Takeuchi, Evidence of a Universal and Isotropic $2 \Delta / k_{B} T_{C}$ Ratio in 122-Type Iron Pnictide Superconductors over a Wide Doping Range, Phys. Rev. B 82, 020515R (2010).

[32] Y. Ji, G. J. Strijkers, F. Y. Yang, C. L. Chien, J. M. Byers, A. Anguelouch, G. Xiao, and A. Gupta, Determination of the Spin Polarization of Half-Metallic $\mathrm{CrO}_{2}$ by Point Contact Andreev Reflection, Phys. Rev. Lett. 86, 5585 (2001).

[33] X. H. Zhang, S. von Molnár, Z. Fisk, and P. Xiong, SpinDependent Electronic States of the Ferromagnetic Semimetal $\mathrm{EuB}_{6}$, Phys. Rev. Lett. 100, 167001 (2008).

[34] H. Z. Arham, C. R. Hunt, W. K. Park, J. Gillett, S. D. Das, S. E. Sebastian, Z. J. Xu, J.S. Wen, Z. W. Lin, Q. Li, G. Gu, A. Thaler, S. Ran, S. L. Bud'ko, P. C. Canfield, D. Y. Chung, M. G. Kanatzidis, and L. H. Greene, Detection of Orbital Fluctuations above the Structural Transition Temperature in the Iron Pnictides and Chalcogenides, Phys. Rev. B 85, 214515 (2012).

[35] See the Supplemental Material at http://link.aps.org/ supplemental/10.1103/PhysRevX.3.011011 for details on crystal characterization, experimental method, and data analysis.

[36] G. Güntherodt, W. A. Thompson, F. Holtzberg, and Z. Fisk, Electron Tunneling into Intermediate-Valence Materials, Phys. Rev. Lett. 49, 1030 (1982).

[37] I. Frankowski and P. Wachter, Point-Contact Spectroscopy on $\mathrm{SmB}_{6}, \mathrm{TmSe}, \mathrm{LaB}_{6}$ and LaSe, Solid State Commun. 41, 577 (1982).
[38] B. Amsler, Z. Fisk, J. L. Sarrao, S. von Molnár, M. W. Meisel, and F. Sharifi, Electron-Tunneling Studies of the Hexaboride Materials $\mathrm{SmB}_{6}, \mathrm{EuB}_{6}, \mathrm{CeB}_{6}$, and $\mathrm{SrB}_{6}$, Phys. Rev. B 57, 8747 (1998).

[39] K. Flachbart, K. Gloos, E. Konovalova, Y. Paderno, M. Reiffers, P. Samuely, and P. Švec, Energy Gap of Intermediate Valent $\mathrm{SmB}_{6}$ Studied by Point-Contact Spectroscopy, Phys. Rev. B 64, 085104 (2001).

[40] D. V. Averin and Yu. V. Nazarov, Virtual ElectronDiffusion During Quantum Tunneling of the Electric Charge, Phys. Rev. Lett. 65, 2446 (1990).

[41] N. E. Sluchanko, V. V. Glushkov, S. V. Demishev, A. A. Pronin, A. A. Volkov, M. V. Kondrin, A. K. Savchenko, and S. Kunii, Low-Temperature Transport Anisotropy and Many-Body Effects in $\mathrm{SmB}_{6}$, Phys. Rev. B 64, 153103 (2001).

[42] S. von Molnár, T. Theis, A. Benoit, A. Briggs, J. Flouquet, J. Ravex, and Z. Fisk in Valence Instabilities, edited by P. Wachter and H. Boppart (North-Holland, Amsterdam, 1982), pp. 389-395.

[43] N. E. Sluchanko, V. V. Glushkov, B. P. Gorshunov, S. V. Demishev, M. V. Kondrin, A. A. Pronin, A. A. Volkov, A. K. Savchenko, G. Grüner, Y. Bruynseraede, V. V. Moshchalkov, and S. Kunii, Intragap States in $\mathrm{SmB}_{6}$, Phys. Rev. B 61, 9906 (2000).

[44] M. Aono, R. Nishitani, C. Oshima, T. Tanaka, E. Bannai, and S. Kawai, $\mathrm{LaB}_{6}$ and $\mathrm{SmB}_{6}$ (001) Surfaces Studied by Angle-Resolved XPS, LEED and ISS, Surf. Sci. 86, 631 (1979).

[45] S. Wolgast, C. Kurdak, K. Sun, J. W. Allen, D.-J. Kim, and Z. Fisk, Discovery of the First Topological Kondo Insulator: Samarium Hexaboride, arXiv:1211.5104.

[46] J. Botimer, D.-J. Kim, S. Thomas, T. Grant, Z. Fisk, and J. Xia, Robust Surface Hall Effect and Nonlocal Transport in $\mathrm{SmB}_{6}$ : Indication for an Ideal Topological Insulator, arXiv:1211.6769. 